\title{
Cartografía inclusiva en la Universidad Eötvös Loránd, Hungría
}

\author{
Ashna Abdulrahman Kareem Zada* \\ Anita Rohonczi* \\ José Jesús Reyes Nuñez*
}

Recibido 08 de abril de 2019; aceptado 10 de junio de 2019

\begin{abstract}
Unfortunately, inclusive cartography does not have a history and tradition as rich in Hungary as in other countries. Beginning our article, the most important cartographic works created for blind and visually impaired people in the country are presented briefly. In recent years, the Department of Cartography and Geoinformatics has decided to make its contribution to the development of this important branch of cartography and special education in Hungary, promoting research projects whose essence lies in finding their own solutions based on international experiences. Two projects are described by the authors: the first one stimulated the renewal of cartographic principles to make maps and atlases of inclusive cartography in Hungary, while the aim of the second project (not concluded yet) is to experiment with these solutions in the autonomous region of Kurdistan in Iraq, beginning the study of the options on the use of the most modern techniques and solutions in the Kurdish special education.

Key words: Inclusive cartography, special education, tactile map, visual disability.

\section{Resumen}

Desafortunadamente, la cartografía inclusiva no cuenta con una historia y tradición tan rica en Hungría como en otros países. Comenzando nuestro artículo se presentan de manera abreviada las obras cartográficas más importantes creadas para personas ciegas y con discapacidad visual en el país. El Departamento de Cartografía y Geoinformática en los últimos años ha decidido hacer su contribución al desarrollo de esta

* Departamento de Cartografía y Geoinformática, Universidad Eötvös Loránd, Hungría, correos electrónicos: ashnakareem88@gmail.com; festyinstyle@gmail.com; jesusreyes@caesar.elte.hu
\end{abstract}


rama tan importante de la cartografía y de la educación especial, promoviendo proyectos de investigación cuya esencia reside en buscar soluciones propias basadas en las experiencias internacionales. Dos proyectos son descritos por los autores: el primero estimula la renovación de los principios cartográficos para hacer mapas y atlas de cartografía inclusiva en Hungría, mientras que el objetivo del segundo proyecto (aún no concluido) es experimentar esas soluciones en la región autónoma de Kurdistán en Irak, iniciando el estudio de las posibilidades de la aplicación de las técnicas y soluciones más modernas en la educación especial kurda.

Palabras clave: Cartografía inclusiva, educación especial, mapa táctil, discapacidad visual.

\section{Resumo}

Infelizmente, a cartografia inclusiva não tem uma história e uma tradição tão ricas na Hungria quanto em outros países. Nosso artigo apresenta de maneira resumida os trabalhos cartográficos mais importantes criados para pessoas com deficiência visual no país. O Departamento de Cartografia e Geoinformática nos últimos anos decidiu contribuir para o desenvolvimento deste importante ramo da cartografia e educação especial, promovendo projetos de pesquisa cuja essência está em encontrar soluções próprias baseadas em experiências internacionais. Dois projetos são descritos pelos autores: o primeiro estimulou a renovação dos princípios cartográficos para fazer mapas e atlas cartográficos inclusivos na Hungria, enquanto o objetivo do segundo projeto (ainda não concluído) é experimentar estas soluções na região autónoma do Curdistão no Iraque, iniciando o estudo das possibilidades de aplicação das técnicas e soluções mais modernas na educação especial curda.

Palavras-chave: Cartografia inclusiva, educação especial, mapa tátil, deficiência visual.

\section{Introducción}

\section{Breve reseña sobre la cartografía inclusiva en Hungría}

A pesar de que la confección de mapas para ciegos y personas con discapacidad visual tiene su origen a mediados del siglo XVIII, a comienzos del siglo XX los maestros que enseñaban en la escuela para alumnos ciegos de Budapest, Hungría, comienzan a hacer los primeros mapas y globos en relieve usando soluciones artesanales. Mapas en relieve, que también podían ser utilizados en la educación especial, fueron hechos en el país desde la segunda mitad del siglo XIX, por ejemplo entre 1872 y 1896 en la sección de producción de mapas de la Imprenta Estatal se elaboraron mapas como "Budapest y sus alrededores" (a escala 1:36 000) y "Sierra Mátra" (1:57 600), pero también podemos mencionar el mapa en relieve del "Lago Balaton y sus alrededores" 
(1:100 000) con un tamaño de 58x88 cm, que fuera publicado para conmemorar los mil años de presencia húngara en la región de los Cárpatos (Klinghammer, 1983).

Después de la Segunda Guerra Mundial, la producción de cartografía inclusiva fue olvidada por largos años y la escuela primaria para ciegos se vió obligada a importar mapas en relieve producidos en la entonces República Democrática Alemana. Tan solo en 1960, fue editado por la empresa estatal Cartographia un mapa en relieve de Hungría a escala de 1:1 250000 y en 1978 otra versión a escala 1:1 000000 , imprimiéndose unos 100 ejemplares de cada uno. En 1983 el Instituto de Cartografía Militar Ágoston Tóth, satisface una solicitud de la Asociación Nacional de Ciegos y Deficientes Visuales y comienza a elaborar mapas en relieve, por ejemplo, un Atlas de Europa y un mapa de Hungría a escala 1:100 000 (Rohonczi, 2007). A estos dos mapas siguió una serie de trece mapas táctiles mostrando la misma cantidad de pasos subterráneos peatonales de Budapest, realizada en 1984 y publicándose 500 ejemplares de cada mapa para el Instituto Nacional de Ciegos y Deficientes Visuales de Hungría (Bíró y Buga, 1985). Todas estas actividades quedaron interrumpidas después de 1989, cuando desaparece el campo socialista.

Actualmente la compañía heredera del Instituto de Cartografía Militar es la única institución estatal del país que cuenta con la tecnología necesaria para producir mapas en relieve, pero debido a los altos costos de producción y el número limitado de usuarios potenciales no se ha continuado trabajando en esa línea. Por esa razón, los maestros del instituto dedicado a la enseñanza de ciegos y deficientes visuales en Budapest, tienen que crear sus propios mapas y globos, o adaptar aquellos que han sido impresos para los alumnos sin problemas visuales. Un ejemplo es el globo que se aprecia en la Figura 1 y que fue adaptado por la maestra de educación especial Brigitta Nagy Lakatosné en el año 2000. Como punto de partida utilizó un globo terrestre impreso para las escuelas húngaras, sobre el que pegó franjas de papel azul para posteriormente redibujar (simplificar) el trazado de las costas de todos los continentes. A continuación, pegó en su superficie representaciones muy generalizadas de las mayores cordilleras (carmelita oscuro) y desiertos (carmelita claro) hechas en cartón, además de ubicar también los paralelos más notables (Ecuador, trópicos y círculos polares), haciéndolos sensibles al tacto con hilos, por lo que el globo puede ser usado tanto por los alumnos ciegos como por deficientes visuales.

Después de muchos años sin contar con una producción profesional de mapas de ese tipo en el país, el Departamento de Cartografía y Geoinformática de la Universidad Eötvös Loránd, decidió comenzar a desarrollar proyectos de investigación relacionados con este tema a partir de la primera década del presente siglo. Simultáneamente empieza también a participar en las actividades programadas por la Comisión de Mapas y Gráficos para Personas Ciegas y con Discapacidad Visual de la Asociación Cartográfica Internacional, para conocer los últimos resultados de las 


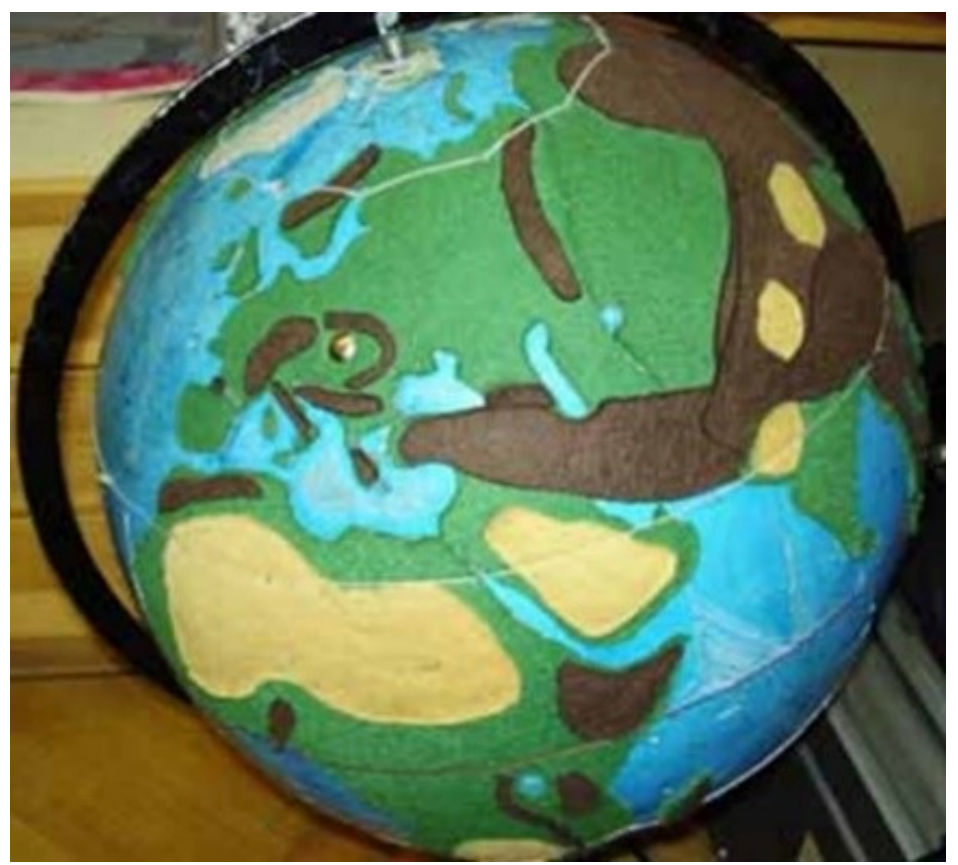

Figura 1. Globo terrestre creado por Brigitta Nagy Lakatosné (2000).

pesquisas científicas y las experiencias obtenidas en otros países. A continuación, se describen dos proyectos del departamento con características muy propias: el primero fue concebido para su implementación dentro del país, el segundo aún está siendo desarrollado para su futuro uso en Irak, específicamente en la región federal autónoma de Kurdistán.

\section{Cartografía inclusiva para alumnos ciegos y con discapacidad visual en Hungría}

Este proyecto fue dividido en dos partes:

- Encuesta sobre la percepción de diferentes tipos de soluciones gráficas usadas en la representación cartográfica y creación de un mapa de Hungría para alumnos ciegos y con discapacidad visual.

- Creación de un atlas escolar para alumnos con discapacidad visual.

\section{Encuesta y creación de un mapa de Hungría}

Primeramente, se elaboraron dos cuestionarios: uno para alumnos ciegos y otro para alumnos con discapacidad visual. En la encuesta participaron nueve alumnos ciegos 
y ocho con discapacidad visual, todos cursaban el 8vo. grado en dos escuelas primarias especiales de la capital, Budapest. La mayoría tenía 14 ó 15 años, tan solo dos alumnos tenían 16 y 18 años de edad respectivamente. Las preguntas de los cuestionarios indagaban sobre las preferencias en diferentes aspectos de la creación de mapas para este grupo específico de usuarios, por ejemplo: qué tipos de líneas y que formas geométricas podían percibir mejor tanto como relleno de un área o como símbolo independiente, mapas de qué tamaños prefieren utilizar y de qué materiales deben estar hechos. También se preguntó sobre los tipos de mapas que conocían y cuáles usaban más a menudo.

Para los alumnos con discapacidad visual se añadió una pregunta para conocer cómo perciben mejor las diferencias de colores en los mapas. Se hicieron cuatro mapas con combinaciones diferentes de colores: intensos, pálidos, combinación de ambos o uso de matices/tonos de un color específico.

El análisis de las respuestas permitió determinar cuatro principios comunes que son necesarios considerar cuando se elabora un mapa tanto para alumnos ciegos como con discapacidad visual:

- Preferencia por figuras geométricas sencillas (círculo, triángulo y cuadrado)

- Uso de líneas continuas al representar el contenido

- Los materiales más aceptados son el papel y el plástico (Figura 2)

- Prefieren usar mapas que pueden sostener cómodamente en sus manos (preferible A3).

Además, las respuestas de los alumnos con discapacidad visual confirmaron que es importante el uso de colores intensos y fuertes contrastes en los mapas.

Considerando los resultados de la encuesta se procedió a crear un mapa temático de Hungría. La idea original era representar datos relacionados con la distribución de personas con discapacidad visual en el país, pero por falta de datos de libre acceso se decidió representar la distribución de la población.

El mapa debería poder ser usado simultáneamente por ciegos y personas con discapacidad visual, cumpliendo con los requisitos por lo menos mínimos para ambos grupos de usuarios y combinando soluciones puramente táctiles con soluciones gráficas para personas con discapacidad visual, como ya se había experimentado en otros países, como por ejemplo en Polonia (Karpinska et al., 2005). Los requisitos mínimos fueron que para representar el contenido temático se combinó el uso de colores intensos con líneas en relieve dibujadas con diferentes orientaciones (horizontal, vertical y diagonal), y que para la nomenclatura del mapa se usó el sistema Braille en conjunto con los nombres escritos con un tipo de letra sencillo (Arial) y con mayúsculas y tamaño de 12 puntos para facilitar su lectura, tratando al mismo tiempo de evitar que ambas soluciones se sobrepusieran una a la otra.

Debido a limitaciones materiales se creó un mapa en formato A4, pero la leyenda se imprimió en una hoja A4 independiente del mapa. Para la impresión del mapa se 


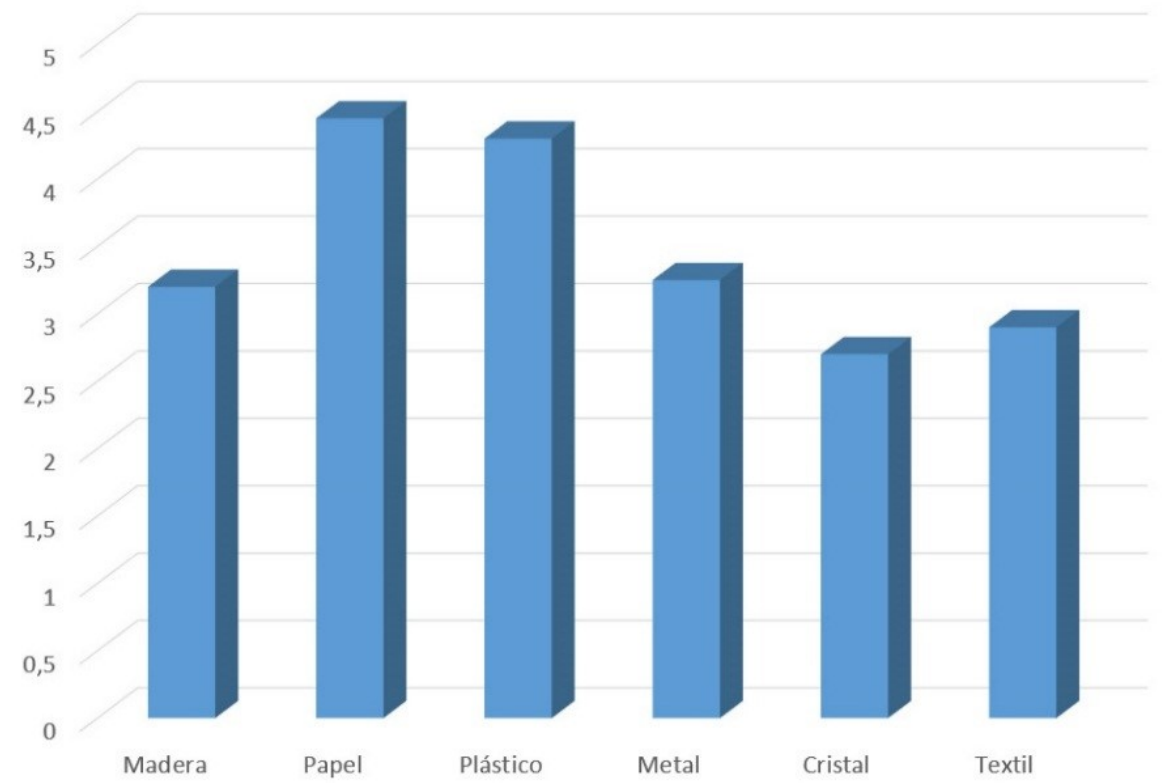

Figura 2. Resultados obtenidos en la encuesta a alumnos ciegos sobre los tipos de materiales preferidos por ellos en los mapas ( 1 -no se pueden percibir las diferencias, 5- se perciben correctamente las diferencias).

usó un equipo ZY-FUSE Heater, que pone de relieve los objetos dibujados en color negro, haciéndolos resaltar sobre la superficie del papel swell usado en este proceso, en el que primero se imprimió de manera tradicional el contenido a color del mapa. El resultado final puede apreciarse en la Figura 3.

\section{Creación de un atlas escolar para alumnos con discapacidad visual}

Este primer trabajo fue seguido por un proyecto dedicado a crear el primer atlas escolar húngaro concebido expresamente para alumnos con discapacidad visual. La investigación fue desarrollada en nuestro departamento y la impresión y publicación del atlas en la empresa privada Cartographia, que se dedica a la publicación de atlas escolares para las escuelas húngaras (Rohonczi, 2007).

En la primera parte del proyecto se determinó el contenido del atlas, para lo cual se estudió el Currículo Nacional Húngaro, ya que la enseñanza en las escuelas especiales del país también es regida por ese Currículo Nacional. Simultáneamente se consultó con los maestros que enseñan Geografía en varias de esas escuelas, para conocer las características particulares de las tareas educativas en esos centros, y cómo son presentados los temas a los alumnos. El título oficial de la obra es Atlas Geográfico Especial para personas con discapacidad visual (Figura 4). 




Figura 3. Mapa temático de Hungría y su leyenda para ciegos y personas con discapacidad visual.

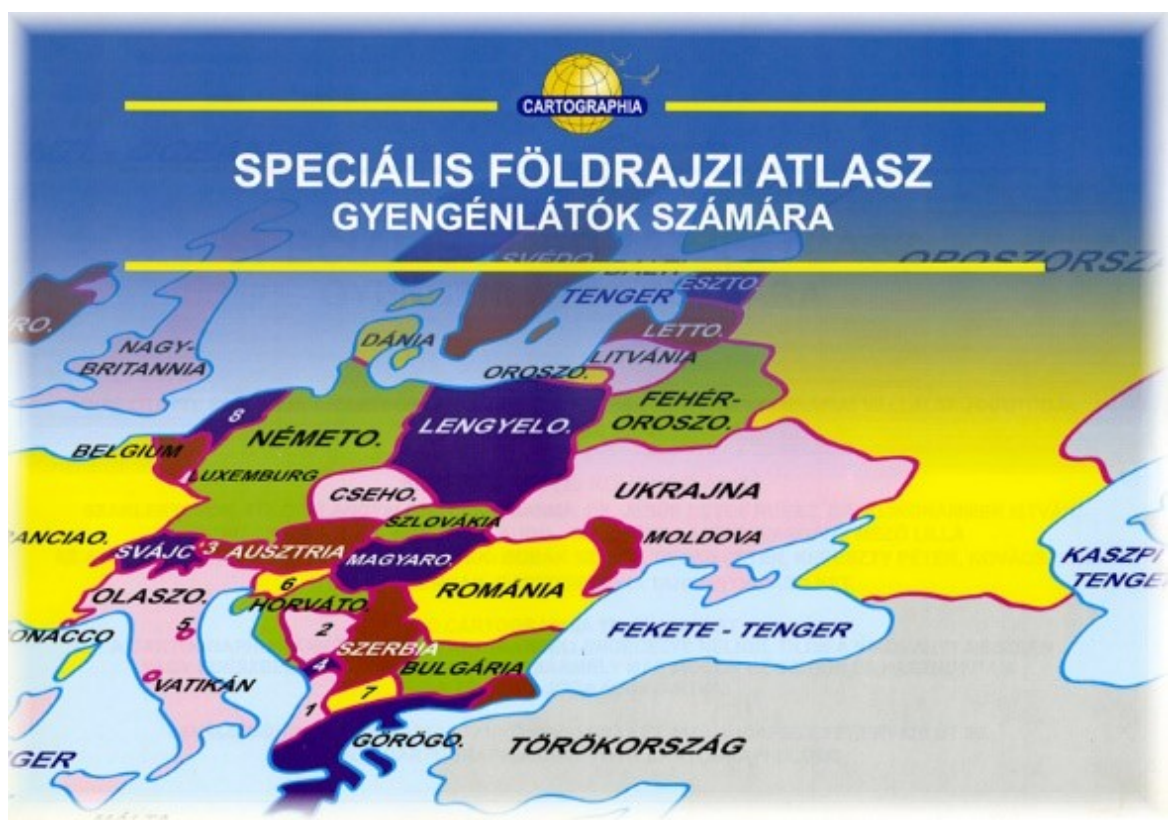

Figura 4. Portada del Atlas Geográfico Especial con un fragmento de un mapa político de Europa. 
Con base a los resultados del estudio del currículo, se decidió que el Atlas estuviera dividido en siete partes, presentando a Hungría, Europa, Asia, África, América, Australia y Oceanía, así como una serie temática de mapas del mundo. El capítulo dedicado a Hungría incluía un mapa físico, uno de recursos minerales y uno político. La presentación de los continentes se hizo con un mapa físico, de recursos minerales, político y de las capitales.

La serie de mapas del mundo incluía: continentes, tectónica de placas, corrientes oceánicas, temperatura media en enero, temperatura media en julio, precipitaciones en enero, precipitaciones en julio, clima, vegetación y husos horarios (Figura 5). El atlas comienza con un mapa del valle de los Cárpatos, como introducción a la presentación del país y termina con una página dedicada a los conocimientos astronómicos.

La segunda parte del proyecto fue dedicada a determinar las características de cada tipo de mapa, cómo representar con una solución gráfica adecuada y eficaz el contenido temático. Para ello se estudiaron algunas experiencias extranjeras, fundamentalmente mapas hechos y usados en países como Brasil (Carmo, 2010) y Polonia (Przyszewska y Szyszkowska, 2011).

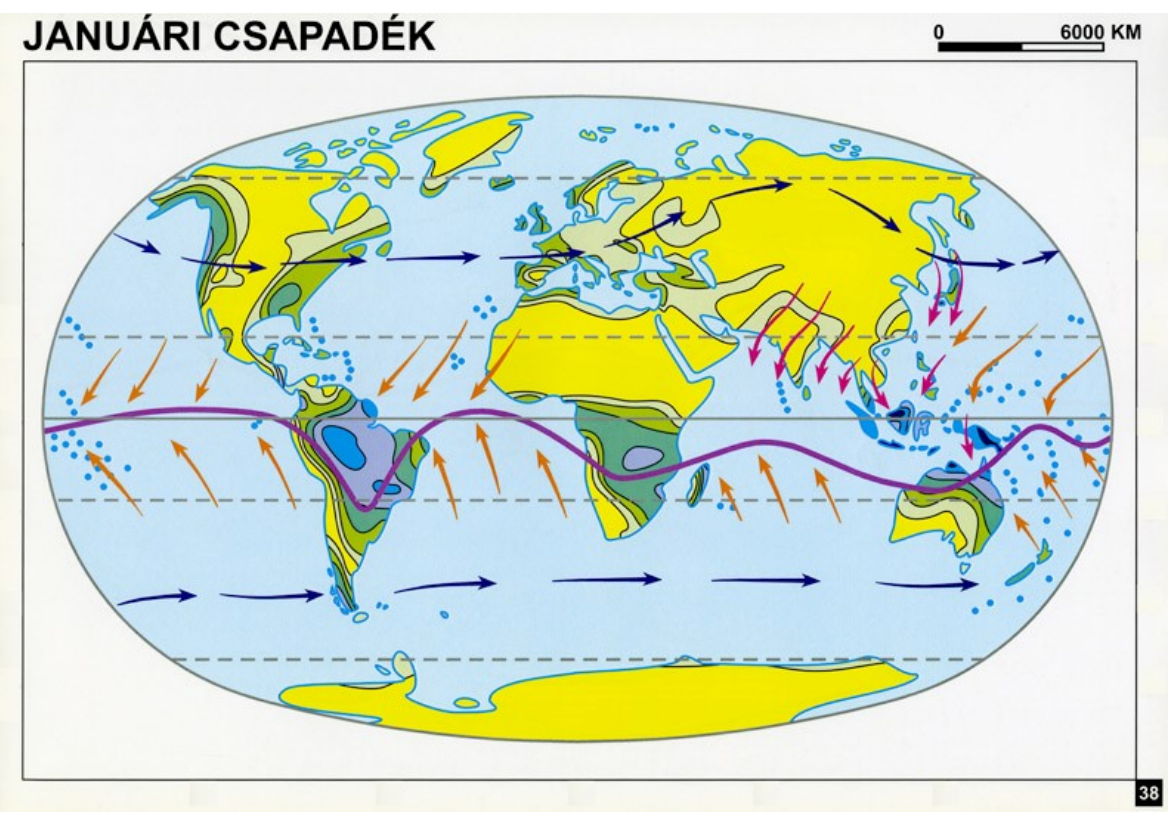

Figura 5. Mapa temático del mundo presentando las precipitaciones en el mes de enero (la leyenda del mapa fue incluida en una página aparte en el mismo atlas). 
Se consideraron y aplicaron también los principios generales determinados en el proyecto anterior (mapa de Hungría), los que fueron complementados con soluciones diseñadas para casos muy específicos en este atlas. Entre ellas podemos mencionar:

- La representación del relieve se hizo muy generalizada, enfatizando con áreas de color intenso la ubicación aproximada de las cadenas montañosas más importantes a nivel continental.

- En lugar de un mapa político de un continente se hicieron dos: uno incluyendo solamente los nombres de los países y otro solo con los nombres de las capitales de cada país. Se adoptó esta solución para evitar una densidad grande de nombres escritos en el mapa, que podría haber dificultado la lectura de los mismos debido al formato del atlas (papel A3) y a la cantidad de nombres geográficos que podían aparecer en un solo mapa (por ejemplo: Europa). Las únicas excepciones fueron América del Sur y del Norte, donde los nombres de los países y las capitales se ubicaron en un solo mapa.

- Los símbolos utilizados en los mapas de recursos minerales están basados en la simbología utilizada por los atlas escolares húngaros, que fueron adaptados a las exigencias propias de un atlas para alumnos con discapacidad visual (mayor tamaño y simplificación de los símbolos manteniendo la figura geométrica original, así como el uso de colores más intensos para los rellenos y de líneas más gruesas para los contornos).

La representación de las islas de la Oceanía se tuvo que hacer a mayor escala que la utilizada en los mapas del mundo, generalizando y enfatizando los contornos para facilitar su identificación por los alumnos. Con el uso de áreas de diferentes colores se representaron las fronteras de cada grupo de islas (Figura 6).

El Atlas fue publicado con una tirada pequeña por primera vez en el año 2009, pero puede ser impreso nuevamente a solicitud de las instituciones o personas interesadas. A finales del 2018 el atlas fue protagonista de una acción posiblemente sin precedentes en otros países: una marca de cosméticos internacionalmente reconocida, en colaboración con la autora y la Asociación Nacional de Personas Ciegas y Deficientes Visuales, ofreció parte de los ingresos obtenidos en la venta de sus productos para donar ejemplares del atlas a escuelas especiales en Hungría (https://hu.loccitane.com/fondation-1-occitane, 16,1,93991,1291985.htm). La campaña de dos semanas de duración tuvo un gran éxito y consiguieron recaudar fondos para comprar 100 ejemplares del atlas y repartirlos gratuitamente entre las escuelas especiales del país. La marca de cosméticos seleccionó esta campaña como la más exitosa organizada por ella en el 2018.

\section{Estudio para el uso de mapas por alumnos con discapacidad visual en Erbil (región autonóma de Kurdistán, Irak)}

Lamentablemente, en los últimos 30 años la región norte de Irak (poblada por miembros de la comunidad kurda) ha estado involucrada o relacionada con diferentes con- 


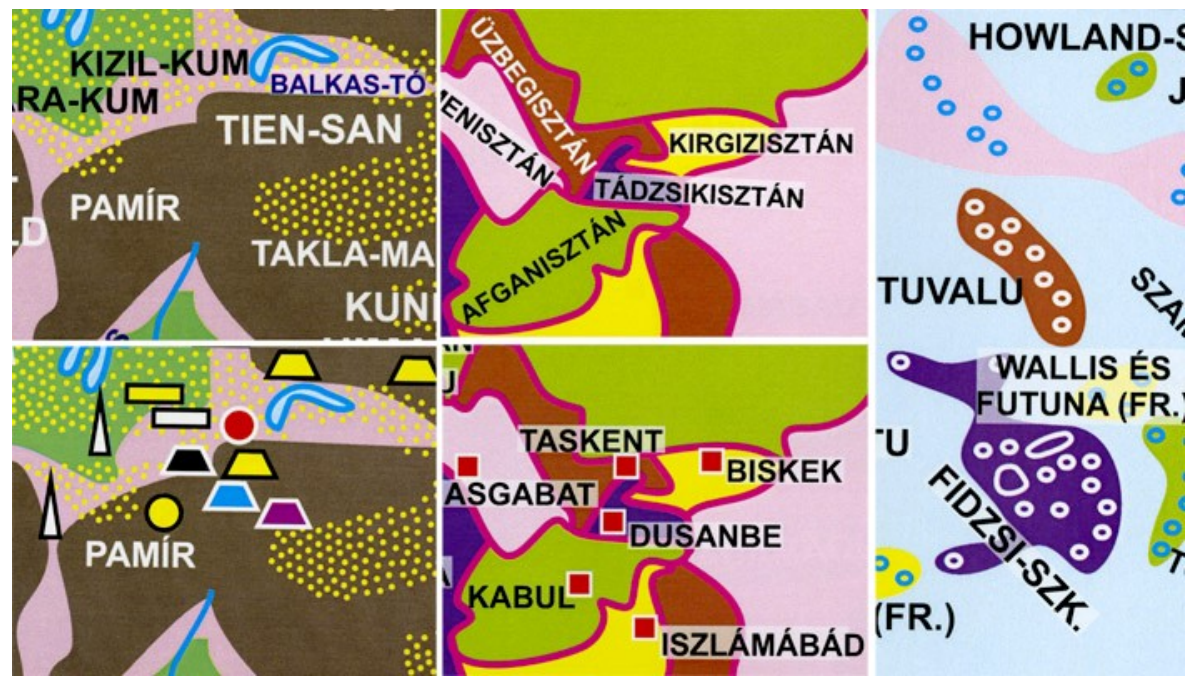

Figura 6. Fragmentos de mapas: en la columna izquierda, representación del relieve y símbolos de los recursos minerales. En la columna central, mapa con nombres de países y con nombres de capitales por separado. En la columna derecha: representación de las islas de Oceanía.

flictos bélicos ocurridos en esa zona, que han tenido una influencia muy negativa en el desarrollo integral de ese territorio. Es por eso que no nos sorprendió conocer que existe una gran falta de mapas y de otros recursos en sus escuelas. Una de las autoras del presente artículo se presentó en nuestro departamento en el año 2016 y manifestó su interés en desarrollar un proyecto de investigación para obtener su grado de doctorado, cuyo tema sería la aplicación en la región de Kurdistán de soluciones modernas usadas en la cartografía para ciegos y personas con discapacidad visual. Comenzó su pesquisa en el 2017 con el estudio de la literatura internacional relacionada con este tema, conociendo aquellas experiencias internacionales que podían ser aplicadas o adaptadas a las condiciones específicas de su país. Esta investigación fue complementada con el análisis del plan de estudios y de los libros de textos usados en la región autónoma de Kurdistán para determinar el contenido temático de los mapas que se crearían durante el proyecto. A continuación, trabajó en la creación de un cuestionario para ser respondido por los alumnos kurdos con discapacidad visual. La encuesta fue desarrollada en el Centro Runaki de la ciudad de Erbil (capital de la región autónoma de Kurdistán en Irak). Esa es una institución establecida en 1990 que atiende las necesidades de las personas ciegas y con discapacidad visual y que cuenta con oficinas en otras ciudades de esa región. El centro está subordinado al Ministerio de Educación y la enseñanza está regida por el mismo plan de estudios que utilizan las escuelas primarias y secundarias del país. En las conversaciones con 
dos maestros de Geografía de ese centro se pudo confirmar la falta de recursos para la educación especial: los alumnos con discapacidad visual creaban sus propios "mapas" de manera totalmente artesanal, usando por ejemplo granos de arroz y frijoles (Figura 7).

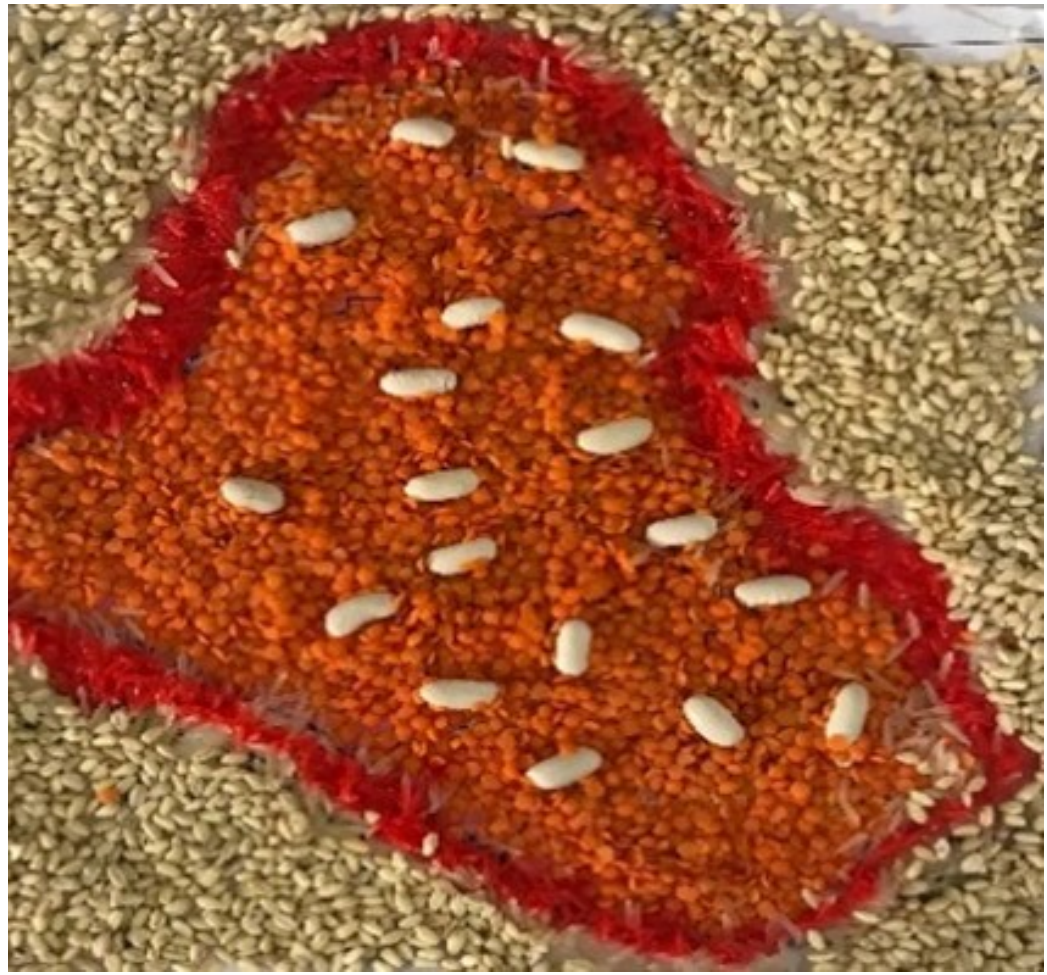

Figura 7. Uno de los mapas artesanales creados por los alumnos del Centro Runaki.

En la encuesta participaron alumnos de nivel primario y secundario, por lo que el intervalo de edad fue muy grande. La edad de los alumnos oscilaba entre seis y nueve años en el nivel primario, y entre nueve 18 años en nivel secundario (Zada, 2019). Participaron 100 alumnos, 64 de nivel primario y 36 de nivel secundario (Figura 8).

El cuestionario estaba formado por 12 preguntas que fueron divididas, en la primera parte se inquiría sobre la experiencia y las preferencias que tenían los alumnos con discapacidad visual usando mapas en sentido general. Según sus respuestas se pudo determinar que: 
- Todos los estudiantes conocían de la existencia de mapas como herramientas de orientación y ubicación geográfica, el $85 \%$ de ellos mostraban interés por los mapas, un 5\% estaba usando un mapa, otro 5\% había usado mapas en la escuela y otro $5 \%$ también fuera de la escuela.

- El 70\% de los alumnos siempre enfrentaban dificultades al usar un mapa impreso en papel y un 30\% tenían dificultades a menudo. Una de las causas de esta situación es que los mapas con que cuentan fueron hechos en otros países y no se adaptan a sus necesidades. En esa región tampoco es posible encontrar aplicaciones programadas en específico para personas con discapacidad visual y existe una falta de computadoras y dispositivos móviles para propiciar el uso de soluciones digitales, por lo que un $80 \%$ de los alumnos expresó que siempre tenía problemas para usar un mapa digital.

- A la pregunta que “¿Cuáles son las razones por las que no había usado un mapa anteriormente? el 50\% de los alumnos adujó la falta de habilidades (conocimientos) suficientes para usarlo, el $31 \%$ la falta de mapas en las escuelas, el 10\% la falta de conciencia de la importancia de usar mapas y tan solo un 5\% consideró que no enfrentó ninguna barrera para usar un mapa, mientras un $4 \%$ adujo otras razones, por ejemplo la falta de equipamiento necesario para imprimir mapas especiales.

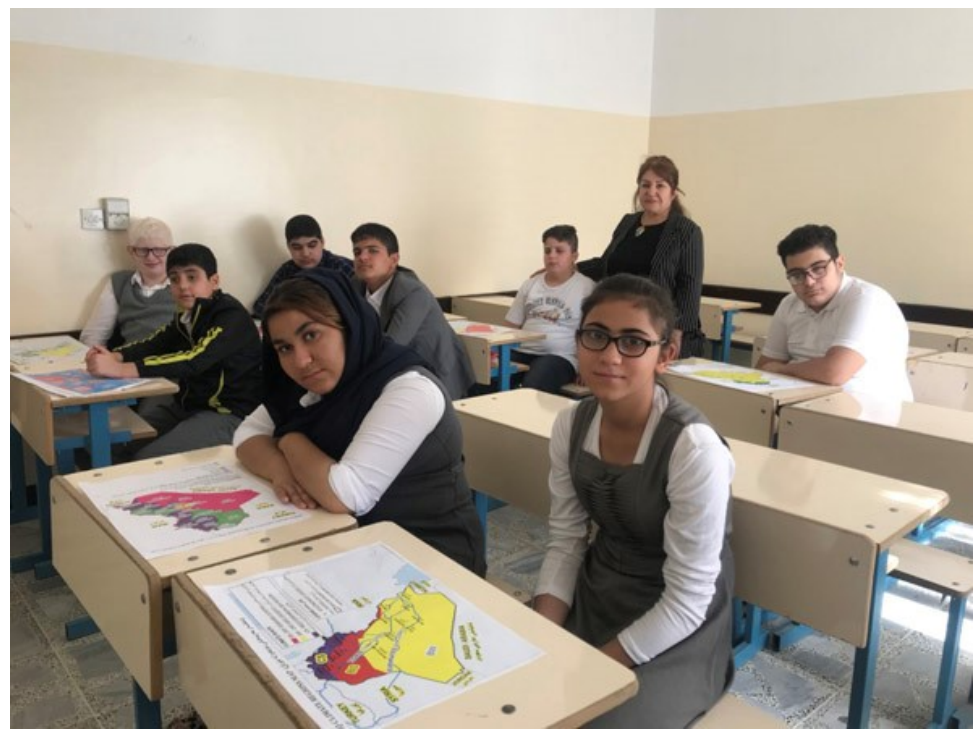

Figura 8. Uno de los grupos de alumnos que participaron en la encuesta.

La segunda parte del cuestionario estaba dedicada a recoger proposiciones para mejorar las soluciones gráficas que se usan en la representación del contenido temático de los mapas en la educación especial. Para ello se mostró una serie de mapas 
creados para esta encuesta, tomando en cuenta las experiencias internacionales en la edición de mapas para alumnos con discapacidad visual y los temas que estudian los alumnos kurdos en las escuelas (Figura 9). Entre las experiencias internacionales que se consultaron merecen destacarse trabajos que han sido desarrollados en Brasil (Vasconcellos, 1993) y Chile (Coll y Pino, 2007). Los mapas fueron hechos usando ArcMap, el módulo de edición de mapas de ArcGIS y fueron impresos a color en papel de formato A3 y A4. Se crearon 11 mapas durante el proyecto: sobre Irak se hizo un mapa administrativo en dos versiones (con fronteras y un solo color, así como de diferentes colores), hidrográfico (solo con los ríos principales), de regiones climáticas, de la distribución de la vegetación, de red de carreteras principales y de la población estimada en el 2016. También se creó un mapa del mundo, un mapa mostrando la ubicación de Irak en el mundo, un mapa administrativo de Irak enfatizando la región de Kurdistán y un mapa del Gran Kurdistán.

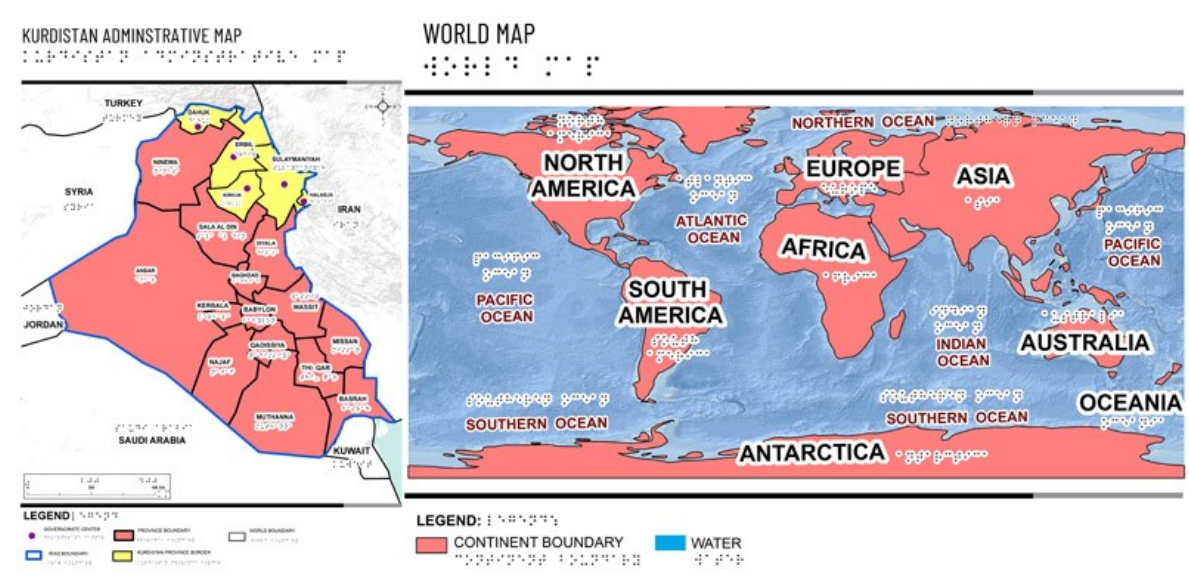

Figura 9. Dos de los mapas hechos especialmente para la encuesta realizada en Erbil.

E1 95\% de los alumnos prefirió usar los mapas en formato A3 y el 80\% consideró que la leyenda usada en los mapas que se mostraba era suficientemente clara para ser usada sin ayuda, aunque se debe reseñar que el $20 \%$ sugirió que la leyenda siempre fuera escrita usando el idioma inglés y el sistema Braille de escritura para ciegos.

Una pregunta esencial fue cuáles colores prefieren que sean usados en los mapas, ofreciendo a elegir entre cuatro opciones: colores claros, colores con fuertes contrastes, una combinación de ellos o en blanco y negro. El 50\% seleccionó la opción de colores con fuertes contrastes, seguida por la combinación de colores con un $40 \%$, mientras que tan solo un $10 \%$ de los alumnos escogía los colores claros y ninguno votó por blanco y negro. 
La respuesta dada a la pregunta sobre las formas geométricas preferidas fue en parte sorpresiva: una inmensa mayoría, el 90\% de los votos recayó en el cuadrado y tan solo el 10\% se decidió por el círculo. Otras formas geométricas (triángulo, estrella y punto) no recibieron ningún voto.

Se incluyó también una pregunta más específica, inquiriendo sobre las formas geométricas que podrían ser utilizadas para diferenciar los límites o fronteras entre áreas (expresado de otra manera: los contornos de los polígonos definidos en el mapa). El 40\% de los alumnos prefirió el cuadrado simple (solo con relleno y sin línea de contorno) y otro $40 \%$ también eligió el cuadrado, pero con su contorno propio. El resto de los alumnos (20\%) votó por líneas onduladas y el posible uso de un gradiente linear.

Finalmente, se preguntó por el tipo de línea que es más fácil distinguir en los mapas y la opinión del $60 \%$ de los participantes coincidió en que la línea continua es más fácilmente reconocible, aunque un $25 \%$ escogía las líneas discontinuas y un $15 \%$ otros tipos de líneas.

Después de las preguntas los alumnos también tenían la oportunidad de escribir sus opiniones personales en relación con los mapas mostrados y sus proposiciones para otros mapas que pueden ser creados en el futuro. Las opiniones dadas fueron positivas, expresando su confianza de que en un futuro puedan usar mapas como esos en las clases. Prácticamente todos también sugirieron que se debería añadir el sistema Braille a los mapas diseñados para la educación especial, incluso si eran destinados a alumnos con discapacidad visual.

En la etapa siguiente de este proyecto se prepararán e imprimirán las versiones de los mapas hechos para alumnos ciegos, que también serán verificados en una encuesta a organizarse en la ciudad de Erbil.

\section{A manera de conclusión}

Durante la investigación relacionada con los alumnos ciegos y con discapacidad visual en Hungría se experimentaron soluciones gráficas, considerando también las respuestas dadas en la encuesta que se organizó en las escuelas especiales de la capital. También se experimentó el uso de una técnica de impresión más económica que las usadas en el pasado, lo que permitió la publicación de un atlas creado específicamente para esas escuelas. Se puede afirmar que los resultados obtenidos durante el proyecto fueron aplicados en la práctica mediante una vía rápida y eficaz, facilitando que el atlas llegara por diferentes vías a manos de los estudiantes con discapacidad visual para su uso diario en las actividades docentes. De manera similar, los mapas creados durante la primera etapa del proyecto que se realiza en Erbil (Irak) demuestran que las experiencias internacionales pueden ser adaptadas con eficacia a las condiciones específicas de un país subdesarrollado y pueden contribuir al uso de nuevas soluciones en la educación especial de esos países. Las respuestas ofrecidas por los alumnos 
kurdos durante la encuesta son una importante contribución para que los mapas futuros sean más adecuados a sus necesidades. Estos resultados motivan al Departamento de Cartografía y Geoinformática de la Universidad Eötvös Loránd a continuar desarrollando las líneas de investigación relacionadas con la cartografía inclusiva en cuatro direcciones fundamentales. La primera es continuar introduciendo en las actividades docentes temas relacionados con la cartografía inclusiva, de manera que nuestros estudiantes puedan conocer más sobre esta área de la cartografía actual. La segunda es impulsar las investigaciones en todos los niveles de la educación superior para desarrollar soluciones novedosas propias y aplicar o adaptar los nuevos resultados alcanzados por investigadores extranjeros no solo en Hungría, sino también en otros países. La tercera es seguir colaborando con la Comisión de Mapas y Gráficos para Personas Ciegas y con Discapacidad Visual de la Asociación Cartográfica Internacional y participando en eventos y otras actividades que programe dicha organización, porque constituye una fuente de referencia para conocer los últimos resultados obtenidos en otros países e intercambiar experiencias con especialistas extranjeros. La cuarta puede considerarse de nivel nacional: estrechar las relaciones con entidades que de una forma u otra están relacionadas con la cartografía inclusiva en Hungría o que pueden jugar un papel protagónico en la difusión y aplicación práctica de los resultados que se obtengan durante las investigaciones.

\section{Bibliografía}

Bíró, J. y Buga, L. (1985). “Tapintással olvasható térképek tervezése és sokszorosítása a vakok számára” (Diseño y reproducción de mapas táctiles para ciegos), en Geodézia és Kartográfia, 5, Budapest, pp. 346-349.

Carmo, W. R. D. (2010). "Cartografia tátil escolar: experiências com a construção de materiais didáticos e com a formação continuada de profesores”. Disertación de Maestría, Faculdade de Filosofia, Letras e Ciências Humanas, Universidad de São Paulo, São Paulo. doi:10.11606/D.8.2010.tde-08032010-124510

Coll E., A. y Pino S., F. (2007). "Impact of tactile cartography on the teaching of Geography in Latin America”, en Proceedings of the $23^{\text {rd }}$ Conference of the International Cartographic Association, 4-10 de agosto, Moscú, Rusia. Recuperado de <https://icaci.org/files/documents/ICC_proceedings/ICC2007/ html/Proceedings.htm $>$.

Karpinska, J., Krauze-Tomczyk, I. y Polak, M. (2005). "Technology of production of maps for the blind and visually handicapped using vector graphics on the protuberant paper", en Proceedings of the $22^{\text {nd }}$ Conference of the International Cartographic Association, 9-16 de junio, A Coruña, España. Recuperado de $<$ https://icaci.org/files/documents/ICC_proceedings/ICC2005/htm/pdf/poster/TE MA22/JUSTYNA\%20KARPINSKA.pdf $>$. 
Klinghammer, I. (1983). "Földünk tükre a térkép" (El mapa, espejo de nuestra Tierra). Editora Gondolat, Budapest, pp. 331.

Przyszewska K., Szyszkowska, K. (2011). "Atlas of the world for the blind and visually impaired -the latest typhlographical publication of Head Office of Geodesy and Cartography on Polish typhlography's background", en Proceedings of the $25^{\text {th }}$ Conference of the International Cartographic Association, 3-8 de julio, París, Francia. Recuperado de <https://icaci.org/files/documents/ICC_proceedings/ ICC2011/Oral\%20Presentations\%20PDF/E3-Mapping\%20for\%20colorblind\% 20or\%20blind\%20users/CO-472.pdf>.

Rohonczi, A. (2007). “Térképszerkesztés vakoknak és gyengénlátóknak: tapasztalatok, javaslatok" (Edición de mapas para ciegos y deficientes visuales: experiencias y recomendaciones), tesis de grado, Universidad Eötvös Loránd, Budapest, $76 \mathrm{pp}$.

Vasconcellos, R. (1993). "Representing the geographical space for visually handicapped students: a case study on map use", en Proceedings of the $16^{\text {th }}$ Conference of the International Cartographic Association, 3-9 de mayo, Colonia, Alemania, pp. 993-1004.

Zada, A.A.K. (2019). "Using Cartography for Partially Impaired People in Kurdistan", en Proceedings of ICC2019, International Cartographic Association (ICA), Tokyo (en proceso de impresión). 